\title{
Measurement and interpretation of the polarization of the $x$-ray line emission of heliumlike Fe XXV excited by an electron beam
}

\author{
P. Beiersdorfer, D. A. Vogel,* K. J. Reed, V. Decaux, J. H. Scofield, and K. Widmann \\ Department of Physics and Space Technology, Lawrence Livermore National Laboratory, Livermore, California 94550 \\ G. Hölzer, E. Förster, and O. Wehrhan \\ Max-Planck Arbeitsgruppe Röntgenoptik, Friedrich-Schiller-Universität, D-07743 Jena, Federal Republic of Germany \\ D. W. Savin \\ Space Science Laboratory, University of California, Berkeley, California 94720 \\ L. Schweikhard \\ Institut für Physik, Johannes Gutenberg Universität, D-55099 Mainz, Federal Republic of Germany
}

(Received 20 November 1995)

\begin{abstract}
The linear polarization of the $1 s 2 p{ }^{1} P_{1} \rightarrow 1 s^{2}{ }^{1} S_{0}$ resonance line, the $1 s 2 p{ }^{3} P_{1,2} \rightarrow 1 s^{2}{ }^{1} S_{0}$ intercombination lines, and the $1 s 2 s^{3} S_{1} \rightarrow 1 s^{2}{ }^{1} S_{0}$ forbidden line was measured in heliumlike Fe XXV excited near threshold by a monoenergetic electron beam. The measurement was carried out with a high-resolution x-ray spectrometer employing a set of two analyzing crystals that acted as polarizers by selectively reflecting the individual polarization components. A value of $+0.56_{-0.08}^{+0.17}$ was determined for the polarization of the ${ }^{1} P_{1}$ line, $-0.53_{-0.02}^{+0.05}$ for the ${ }^{3} P_{2}$ line, $-0.22_{-0.02}^{+0.05}$ for the ${ }^{3} P_{1}$ line, and $-0.076_{-0.007}^{+0.007}$ for the ${ }^{3} S_{1}$ line. The measurements were compared with results from a relativistic distorted-wave calculation, which was carried out for a number of mid- $Z$ heliumlike ions $\left(\mathrm{Mg}^{10+}-\mathrm{Kr}^{3++}\right)$, and good agreement was found. By contrast, disagreement was noted with predictions based on Coulomb-Born calculations, allowing us to distinguish between theoretical approaches. [S1050-2947(96)05406-6]
\end{abstract}

PACS number(s): 32.30.Rj, 32.70.Fw, 34.80.Kw, 95.30.Ky

\section{INTRODUCTION}

Quasistationary ions colliding with unidirectional electrons generally emit line radiation that is both anisotropic and polarized $[1,2]$. The intensity of a specific line from a beam-excited ion depends on the observation angle relative to the axis defined by the electron beam and on whether or not the emission is analyzed with a polarization-sensitive instrument.

Polarized x-ray emission has been observed from a variety of plasma sources, such as vacuum sparks or laserproduced plasmas [3-5]. Here, the observation of polarized $\mathrm{x}$-ray emission serves as a diagnostic marker of the presence of directional electrons in the source. Excitation by directional electrons has also been implicated in the production of anomalous line intensities observed in spectra from solar flares [6,7]. Proper accounting of the effects of anisotropy and polarization resulting from beam excitation is a prerequisite for employing x-ray line intensities for such diagnostic purposes.

In virtually all cases where excitation by an electron beam plays a role, theoretical predictions have been used to aid the analysis of the observations. Tests of these predictions have been made in only a few cases. A measurement of the anisotropy or polarization of the x-ray emission from beamexcited highly charged heliumlike ions has been reported to

\footnotetext{
*Present address: HQ ACC/DRAN, Langley Air Force Base, VA 23665 .
}

our knowledge only for transitions in heliumlike Sc XX [8]. This measurement was performed on the Livermore electronbeam ion trap (EBIT) facility $[9,10]$. In the following we use the same facility to measure the polarization of the electronbeam-excited x-ray line emission from heliumlike Fe XXV. Unlike the isotopes of scandium, the most abundant isotopes of iron $(>98 \%)$ lack a nuclear magnetic moment. As a result, the hyperfine interaction plays no role in determining the polarization of the beam-excited x-ray lines, and the polarization of several of the heliumlike Fe XXV lines differs markedly from that of heliumlike Sc XX. In the latter ion, the hyperfine interaction virtually eliminates beam-induced polarization effects for transitions from the triplet levels. The present measurement thus tests theory in a different regime than the scandium results. Since most recent calculations have been performed for heliumlike ions where the hyperfine interaction is absent [11-14], such a test is especially important.

The present measurement relies on the use of two analyzing crystals with different lattice spacings. The technique requires knowledge of the reflection parameters of the crystals [15], and calculations of these parameters are presented. The technique, in principle, also requires the presence of an unpolarized line for cross normalization. An appropriate unpolarized line does not exist in the spectrum of Fe XXV. We show that cross normalization of the relative line intensities can be accomplished by making use of the fact that the polarization of the $1 s 2 s{ }^{3} S_{1} \rightarrow 1 s^{2}{ }^{1} S_{0}$ forbidden line, labeled $z$ in standard notation [16], is linked in a simple way to the polarization of the $1 s 2 p^{3} P_{2} \rightarrow 1 s^{2}{ }^{1} S_{0}$ intercombination 
line, labeled $x$. Based on this observation, a formalism is developed for interpreting our data that is generally applicable to polarization measurements of heliumlike ions and may be used in measurements along the isoelectronic sequence.

In order to interpret the observed polarizations, we performed distorted-wave calculations over a range of mid- $Z$ elements (from neon to krypton). These indicate that the polarization of line $x$ remains virtually unchanged. This is similar to the behavior of the polarization of the $1 s 2 p{ }^{1} P_{1} \rightarrow 1 s^{2}{ }^{1} S_{0}$ resonance line, labeled $w$, which had been shown earlier to remain essentially unchanged as a function of $Z$ [12]. A slight variation occurs only because of relativistic effects [14]. The polarization of line $z$ vanishes in the absence of radiative cascades. A finite value is attained because of radiative cascades from the ${ }^{3} P_{2}$ level. The polarization of $z$, thus, would remain essentially fixed as well, except for the fact that the amount of the radiative cascade contributions from the ${ }^{3} P_{2}$ level varies strongly as a function of atomic number [17]. The finite value of the polarization for line $z$ thus reflects the population fraction received from the ${ }^{3} P_{2}$ level. By contrast, the polarization of the $1 s 2 p{ }^{3} P_{1} \rightarrow 1 s^{2}{ }^{1} S_{0}$ intercombination line, labeled $y$, exhibits a strong variation over the range of elements considered, which results from the increasing importance of relativistic effects as $Z$ increases. A comparison of these calculations with the measured values for Fe XXV shows agreement well within the experimental uncertainties, validating the results of the distorted-wave calculation. By contrast, our measurement disagrees in part with the results of an earlier calculation using the Coulomb-Born approximation without exchange $[18,19]$.

\section{THEORY}

In general, the intensity and polarization of a given line from a beam-excited ion depends on the observation angle relative to the direction defined by the relative velocities of the ion and electron. We assume quasistationary ions colliding with monoenergetic electrons traveling in the $z$ direction. Then, it is common to define the linear polarization $P$ at an observation angle $\vartheta=90^{\circ}$ relative to the $z$ axis pointing in the direction of the electron beam as the fractional difference between the intensity of light with electric field vector parallel to the beam direction, $I_{\|}$, and the intensity of light with electric field vector perpendicular, $I_{\perp}[2]$ :

$$
P=\frac{I_{\|}-I_{\perp}}{I_{\|}+I_{\perp}}
$$

Following the prescription by Steffen and Alder [20] the two intensity components of linearly polarized line radiation from a cylindrically symmetric source emitting multipole radiation described by a single multipole operator are given by

$$
I_{\|}=\frac{1}{2} \sum_{\lambda=\text { even }} B_{\lambda} A_{\lambda}\left[P_{\lambda}(0)+\Gamma(\kappa) f_{\lambda} P_{\lambda}^{2}(0)\right] I_{s}
$$

$$
I_{\perp}=\frac{1}{2} \sum_{\lambda=\text { even }} B_{\lambda} A_{\lambda}\left[P_{\lambda}(0)-\Gamma(\kappa) f_{\lambda} P_{\lambda}^{2}(0)\right] I_{s} .
$$

Here, $B_{\lambda}$ is the orientation parameter, $A_{\lambda}$ is the angular distribution coefficient, and the product $A_{\lambda} \Gamma(\kappa) f_{\lambda}$ describes the linear polarization parameter. $P_{\lambda}(0)$ and $P_{\lambda}^{2}(0)$ represent the Legendre and associated Legendre polynominal, respectively, evaluated at the origin, and $\lambda$ is the multipole order of the emitted radiation. $I_{s}$ is the ion line source intensity.

The parameters $B_{\lambda}$ describe the initial state of the system and are given by

$$
\begin{aligned}
B_{\lambda}= & \sum_{m}(-1)^{J_{i}+m}\left[(2 \lambda+1)\left(2 J_{i}+1\right)\right]^{1 / 2} \\
& \times\left(\begin{array}{ccc}
J_{i} & J_{i} & \lambda \\
-m & m & 0
\end{array}\right) \sigma_{m} .
\end{aligned}
$$

Here $J_{i}$ is the initial total angular momentum, $m$ is the magnetic quantum number, and $\sigma_{m}$ is the population density of each respective sublevel normalized such that $\Sigma_{m} \sigma_{m}=1$. The quantity in large parentheses denotes the Wigner 3-j symbol. Under our assumption that the emitted photons are described by a single multipole operator, the angular distribution coefficient $A_{\lambda}$ is given by

$$
\begin{aligned}
A_{\lambda}= & (-1)^{J_{i}+J_{f}-1}\left[(2 \lambda+1)\left(2 J_{i}+1\right)\right]^{1 / 2}(2 L+1) \\
& \times\left(\begin{array}{ccc}
L & L & \lambda \\
1 & -1 & 0
\end{array}\right)\left\{\begin{array}{ccc}
L & L & \lambda \\
J_{i} & J_{i} & J_{f}
\end{array}\right\},
\end{aligned}
$$

where $L$ gives the order of what Steffen and Alder call pure $2^{L}$ decays. The quantity in large braces denotes the Wigner 6- $j$ symbol. In highly charged heliumlike ions, the lines observed are from the "allowed" electric dipole $(E 1)$ transitions $w$ and $y$ with $L=1$ as well as from the "forbidden" magnetic dipole $(M 1)$ transition $z$ and the magnetic quadrupole (M2) transition $x$ and $L=1$ and 2 , respectively. The coefficient $f_{\lambda}$ is given by

$$
f_{\lambda}=-\left[\frac{(\lambda-2) !}{(\lambda+2) !}\right]^{1 / 2} \frac{\left(\begin{array}{ccc}
L & L & \lambda \\
1 & 1 & -2
\end{array}\right)}{\left(\begin{array}{ccc}
L & L & \lambda \\
1 & -1 & 0
\end{array}\right)} .
$$

The function $\Gamma(\kappa)$ determines the sign. For electric multipole transitions it equals $\Gamma(E)=1$, for magnetic multipole transitions it equals $\Gamma(M)=-1$.

Substituting Eqs. (2) and (3) the polarization is given by

$$
P=\Gamma(\kappa) \frac{\Sigma_{\lambda=\mathrm{even}} B_{\lambda} A_{\lambda} f_{\lambda} P_{\lambda}^{2}(0)}{\sum_{\lambda=\mathrm{even}} B_{\lambda} A_{\lambda} P_{\lambda}(0)} .
$$

Through $B_{\lambda}$ the value of the polarization of a given line is linked to the population densities $\sigma_{m}$ of the magnetic sublevels. Evaluating the coefficients, the polarization of the electric dipole lines $w$ and $y$ is described by

$$
P=-\frac{\sigma_{-1}-2 \sigma_{0}+\sigma_{+1}}{\sigma_{-1}+2 \sigma_{0}+\sigma_{+1}} .
$$


TABLE I. Total cross section $\sigma_{\text {total }}$ and fractional population density $\sigma_{m}$ of each magnetic sublevel $m$ for electron-impact excitation of a given level from the $1 s^{2}{ }^{1} S_{0}$ ground state of heliumlike Fe XXV at an electron energy of $6800 \mathrm{eV}$. Numbers in brackets denote powers of ten.

\begin{tabular}{lcccc}
\hline \hline Level & $\begin{array}{c}\sigma_{\text {total }} \\
\left(\mathrm{cm}^{2}\right)\end{array}$ & $\sigma_{0}$ & $\sigma_{ \pm 1}$ & $\sigma_{ \pm 2}$ \\
\hline $1 s 2 p{ }^{1} P_{1}$ & $3.75[-22]$ & 0.655 & 0.167 & \\
$1 s 2 p{ }^{3} P_{0}$ & $3.91[-23]$ & 1.000 & & \\
$1 s 2 p{ }^{3} P_{1}$ & $1.38[-22]$ & 0.253 & 0.373 & \\
$1 s 2 p{ }^{3} P_{2}$ & $1.84[-22]$ & 0.316 & 0.259 & 0.083 \\
$1 s 2 s{ }^{1} S_{0}$ & $1.35[-22]$ & 1.000 & & \\
$1 s 2 s{ }^{3} S_{1}$ & $6.26[-23]$ & 0.333 & 0.333 & \\
\hline \hline
\end{tabular}

$$
P=\frac{\sigma_{-1}-2 \sigma_{0}+\sigma_{+1}}{\sigma_{-1}+2 \sigma_{0}+\sigma_{+1}},
$$

while for line $x$, it is

$$
P=\frac{\sigma_{-2}-\sigma_{-1}-\sigma_{+1}+\sigma_{+2}}{\sigma_{-2}+\sigma_{-1}+\sigma_{+1}+\sigma_{+2}} .
$$

Since the electron beam is unpolarized, $\sigma_{m}=\sigma_{-m}$.

The values of $\sigma_{m}$ for excitation of heliumlike iron have been calculated by Zhang, Sampson, and Clark with a fully relativistic distorted-wave computer code for electron energies of 7.5-27 keV [21]. Using the same computer code we calculated the values of $\sigma_{m}$ near threshold $(6.8 \mathrm{keV})$. The results are given in Table I for Fe XXV. From these values we compute $P_{w}=0.599, P_{y}=-0.192, P_{x}=-0.515$, and $P_{z}=0$, as summarized in Table II. These values are close to the values computed by Inal and Dubau [11], who also used a distorted-wave computer code for calculating the excitation cross sections of the magnetic sublevels. They differ, however, from the values reported by Vinogradov, Urnov, and Shlyaptseva computed from Coulomb-Born cross sections $[18,19]$. For lines $w$ and $x$ the difference is more than $20 \%$, as seen from Table II.

Because line $z$ is populated by cascades from higher-lying levels, knowing the values of $\sigma_{m}$ for electron-impact excitation of the ${ }^{3} S_{1}$ level from the ground state is insufficient to predict the actual polarization of the line. According to the calculations by Lin, Johnson, and Dalgarno [17] the $1 s 2 p{ }^{3} P_{2}$ level in Fe XXV decays via emission of line $x$ only
$81.7 \%$ of the time. The rest feeds the $1 s 2 s{ }^{3} S_{1}$ level. Moreover, in the absence of a nuclear magnetic moment the $1 s 2 p{ }^{3} P_{0}$ level decays $100 \%$ of the time to the $1 s 2 s{ }^{3} S_{1}$ level. The effect of these cascade contributions on the sublevel populations needs to be considered for accurately predicting the polarization of line $z$.

As discussed by Steffen and Alder [20], the effects of cascades can be described by the deorientation factor $U_{\lambda}$,

$$
U_{\lambda}=(-1)^{J_{i}+J_{f}+\lambda+L}\left[\left(2 J_{i}+1\right)\left(2 J_{f}+1\right)\right]^{1 / 2}\left\{\begin{array}{lll}
J_{i} & J_{i} & \lambda \\
J_{f} & J_{f} & L
\end{array}\right\} .
$$

Given the orientation parameter $B_{\lambda}(|i\rangle)$ of the initial state $|i\rangle$, the orientation parameter associated with populating the state $|f\rangle$ by the cascade can then be expressed as

$$
B_{\lambda}(|f\rangle)=B_{\lambda}(|i\rangle) U_{\lambda}\left(J_{i}, J_{f}, L\right) .
$$

The orientation parameter of a level populated by various direct and indirect processes is then given by the weighted sum of the orientation parameters associated with each process contributing to its population,

$$
B_{\lambda}(|f\rangle)=\sum_{i} f_{i} B_{\lambda}(|i\rangle) U_{\lambda}\left(J_{i}, J_{f}, L\right)
$$

where $f_{i}$ is the fraction of the population received from process $i$.

Evaluating the coefficients we find $U_{2}(2,1,1)=\sqrt{7 / 20}$ for the deorientation parameter for cascades from the level $1 s 2 p{ }^{3} P_{2}$ to $1 s 2 s{ }^{3} S_{1}$. Moreover, $B_{2}=0$ for excitation of the ${ }^{3} S_{1}$ level from the ground state as well as for radiative cascades from the ${ }^{3} P_{0}$ level. Hence the orientation parameter of the ${ }^{3} S_{1}$ level populated by radiative cascades from the ${ }^{3} P_{0}$ and ${ }^{3} P_{2}$ levels and by direct electron-impact excitation from the ground state can be expressed as

$$
\begin{aligned}
B_{2}\left({ }^{3} S_{1}\right)= & \left(\frac{7}{20}\right)^{1 / 2} \frac{\beta_{r} \sigma_{\text {total }}\left({ }^{3} P_{2}\right)}{\sigma_{\text {total }}\left({ }^{3} S_{1}\right)+\sigma_{\text {total }}\left({ }^{3} P_{0}\right)+\beta_{r} \sigma_{\text {total }}\left({ }^{3} P_{2}\right)} \\
& \times B_{2}\left({ }^{3} P_{2}\right) .
\end{aligned}
$$

Here $\beta_{r}=0.183$ is the branching ratio for ${ }^{3} P_{2} \rightarrow{ }^{3} S_{1}$ decay, and $\sigma_{\text {total }}$ denotes the total excitation cross section for the respective levels given in Table I. Inserting the values from Table I, we obtain $B_{2}\left({ }^{3} P_{2}\right)=-0.49$ so that

TABLE II. Comparison of calculated and measured values of the polarization of lines $w, x, y$, and $z$ at an excitation energy of $6800 \mathrm{eV}$.

\begin{tabular}{lcccc}
\hline \hline Line & $\begin{array}{c}\text { Shlyaptseva } \\
\text { and co-workers }^{\mathrm{a}}\end{array}$ & $\begin{array}{c}\text { Inal and } \\
\text { Dubau }\end{array}$ & $\begin{array}{c}\text { Present } \\
\text { calculations }\end{array}$ & Measurements \\
\hline$P_{w}$ & +0.82 & +0.584 & +0.599 & $+0.56_{-0.08}^{+0.17}$ \\
$P_{x}$ & -0.75 & -0.518 & -0.515 & $-0.53_{-0.02}^{+0.05}$ \\
$P_{y}$ & -0.23 & -0.196 & -0.192 & $-0.22_{-0.02}^{+0.05}$ \\
$P_{x}$ (no cascades) & 0.000 & 0.000 & 0.000 & \\
$P_{z}$ (with cascades) & & -0.078 & -0.074 & $-0.076_{-0.007}^{+0.007}$ \\
\hline \hline
\end{tabular}

${ }^{\mathrm{a}}$ References [18,19].

${ }^{\mathrm{b}}$ References $[11,13]$. 
$B_{2}\left({ }^{3} S_{1}\right)=-0.072$. Substituting this value into Eq. (7) yields $P_{z}=-0.074$ for the polarization of line $z$. The value is in good agreement with the value of -0.078 calculated recently by Inal and Dubau [13].

Looking at Eq. (14) we note that $B_{2}\left({ }^{3} S_{1}\right)$ and thus $P_{z}$ depends only on $B_{2}\left({ }^{3} P_{2}\right)$ and on the fraction $k$ given by

$$
k=\left(\frac{7}{20}\right)^{1 / 2} \frac{\beta_{r} \sigma_{\text {total }}\left({ }^{3} P_{2}\right)}{\sigma_{\text {total }}\left({ }^{3} S_{1}\right)+\sigma_{\text {total }}\left({ }^{3} P_{0}\right)+\beta_{r} \sigma_{\text {total }}\left({ }^{3} P_{2}\right)} .
$$

To determine how $B_{2}\left({ }^{3} S_{1}\right)$ and the individual terms in $k$ vary with atomic number, we have performed distorted-wave calculations of the magnetic sublevel populations near threshold over a range of elements from neon $(Z=10)$ to krypton $(Z=36)$. We find that $B_{2}\left({ }^{3} P_{2}\right)$ essentially remains constant at a value of -0.49 . As a result, $P_{x}$ remains virtually constant, changing merely from -0.525 for Ne IX to -0.511 for $\mathrm{Kr}$ XXXV. Moreover, the excitation cross section for populating the ${ }^{3} P_{2}$ level remains essentially constant relative to that for populating the ${ }^{3} P_{0}$ and ${ }^{3} S_{1}$ levels. In particular, the ratio $\sigma_{\text {total }}\left({ }^{3} P_{2}\right) /\left[\sigma_{\text {total }}\left({ }^{3} S_{1}\right)+\sigma_{\text {total }}\left({ }^{3} P_{0}\right)+\sigma_{\text {total }}\left({ }^{3} P_{2}\right)\right]$ drops merely from 0.66 for Ne IX to 0.63 for Kr XXXV. The polarization of line $z$ thus is essentially independent of the magnetic sublevel populations and depends only on the radiative branching ratio $\beta_{r}$. This ratio has been calculated for most elements by Lin, Johnson, and Dalgarno [17]. It varies strongly from element to element, dropping from 0.98 for Ne IX to 0.10 for Kr XXXV. We compute a corresponding change of the polarization of $z$ from -0.187 to -0.049 , respectively.

The dependence of the polarization of line $z$ on cascade contributions from the ${ }^{3} P_{2}$ level can also be expressed directly in a simple relationship between the polarizations $P_{z}$ and $P_{x}$. To do so we note that the orientation parameter $B_{4}\left({ }^{3} P_{2}\right) \ll B_{2}\left({ }^{3} P_{2}\right)$. In particular, employing the sublevel population densities given in Table $I$, we find $B_{4}\left({ }^{3} P_{2}\right) / B_{2}\left({ }^{3} P_{2}\right) \approx 1.6 \times 10^{-3}$. Similar ratios are found for other heliumlike ions. We can thus set

$$
P_{x}=-\frac{B_{2}\left({ }^{3} P_{2}\right) A_{\lambda} f_{2} P_{2}^{2}(0)}{\sum_{\lambda=\operatorname{even}} B_{\lambda}\left({ }^{3} P_{2}\right) A_{\lambda} P_{\lambda}(0)} .
$$

Evaluating the coefficients and solving for $B_{2}\left({ }^{3} P_{2}\right)$ we get

$$
B_{2}\left({ }^{3} P_{2}\right)=+\left(\frac{14}{5}\right)^{1 / 2} \frac{2 P_{x}}{3-P_{x}}
$$

The expression for $P_{z}$ can then be expressed in terms of $P_{x}$ as

$$
P_{z}=+\frac{3 k B_{2}\left({ }^{3} P_{2}\right)}{2 \sqrt{2}-k B_{2}\left({ }^{3} P_{2}\right)}
$$

Here, the factor $k$ is given by Eq. (15). Substituting the expression in Eq. (17) for $B_{2}\left({ }^{3} P_{2}\right)$ into Eq. (18) yields a direct expression for $P_{z}$ in terms of $P_{x}$ :

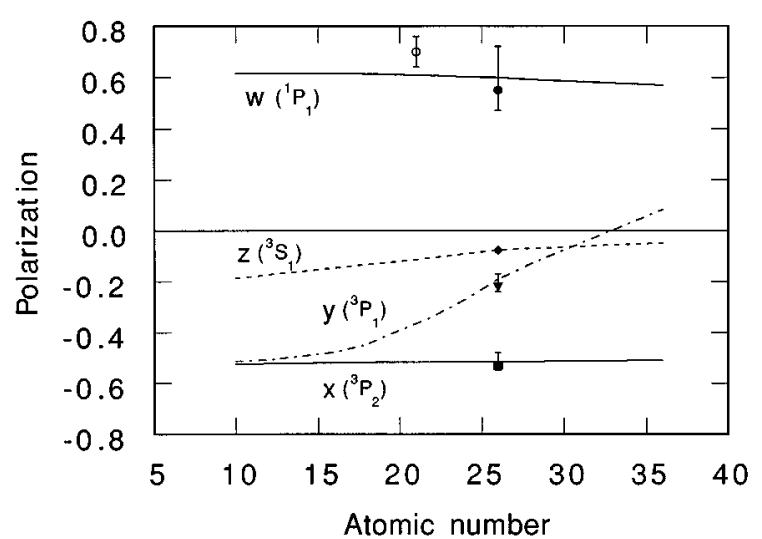

FIG. 1. Predicted polarizations of the resonance line $w$, the intercombination lines $x$ and $y$, and the forbidden line $z$ in heliumlike ions between Ne IX and Kr XXXV. The predictions are for nuclei without a magnetic moment. Strong hyperfine interaction with the nucleus results in vanishing polarizations for the triplet lines. The values measured for Fe XXV are shown for comparison. Also shown is the measured value for the singlet line in Sc XX (open circle) from Ref. [8].

$$
P_{z}=+\frac{3 k \sqrt{\frac{14}{5}} P_{x}}{3 \sqrt{2}-P_{x}\left(\sqrt{2}+k \sqrt{\frac{14}{5}}\right)} .
$$

This simple relationship between the polarization of lines $x$ and $z$ reduces the number of unknown parameters in a given measurement. The relationship forms one of the cornerstones of the experimental procedure developed in the next section.

An overview of the change in the calculated polarizations of the four heliumlike lines is given in Fig. 1. As discussed, the polarization of $x$ is virtually constant. The same is true for the resonance line $w$. The polarization of $z$ varies because of the differing fraction of radiative cascade contributions populating its upper level. The polarization of $y$ varies even more strongly than that of line $z$. This reflects the strong influence of relativity. Its polarization is essentially equal to that of $x$ at low $Z$; it approaches that of $w$ for high $Z$.

\section{EXPERIMENTAL PROCEDURE}

The reflectivity of an analyzing crystal depends on the polarization component. The intensity of an x-ray line observed with a crystal spectrometer is

$$
I^{\mathrm{obs}}=R_{\|} I_{\|}+R_{\perp} I_{\perp},
$$

where $R_{\|}$and $R_{\perp}$ are the integrated crystal reflectivities for $\mathrm{x}$ rays polarized perpendicular and parallel to the plane of dispersion. Because generally $R_{\|} \neq R_{\perp}$ crystal spectrometers can be used as polarimeters to determine the polarization of emitted light.

The ratio $R=R_{\perp} / R_{\|}$depends strongly on the Bragg angle $\theta$. For mosaic crystals the ratio varies as $\cos ^{2}(2 \theta)$; for perfect crystals it varies as $|\cos (2 \theta)|[22]$. The ratio for actual crystals lies in between the two extremes. The experimental procedure used in the following is based on the fact that measuring a set of x-ray lines at two differing Bragg angles 


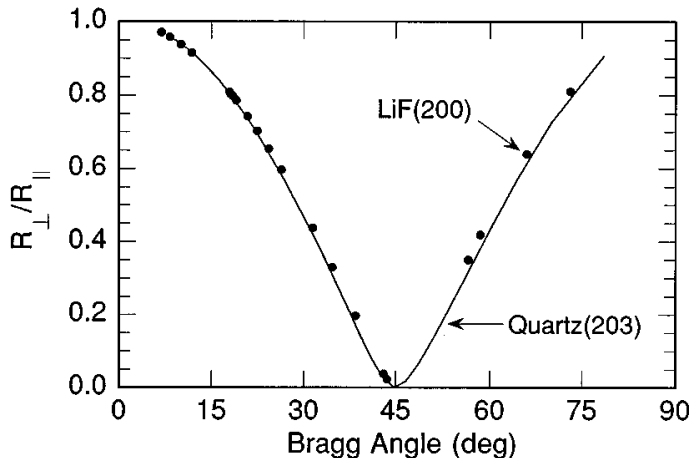

FIG. 2. Variation of the relative crystal reflectivities $R_{\perp} / R_{\|}$for $\mathrm{x}$ rays polarized perpendicular and parallel to the electron-beam direction. The values for $\operatorname{LiF}(200)$ are calculated values from Ref. [23] (solid circles); those for Quartz(203) are present calculations (solid line).

allows us, in principle, to determine the two intensity components $I_{\|}$and $I_{\perp}$ provided $R(\theta)$ is known. For such a measurement analyzing crystals with differing lattice spacing $2 d$ are needed. In our measurements we used Quartz(203), which has a lattice spacing $2 d=2.750 \AA$, and $\operatorname{LiF}(200)$ with $2 d=4.027 \AA$; the corresponding observation angles are $\theta=42.5^{\circ}$ and $27.5^{\circ}$, respectively, for the $K \alpha$ transitions of heliumlike Fe XXV.

Calculations of integrated reflectivities of perfect crystals were recently presented by Henke, Gullikson, and Davis [23]. These calculations include those for $\operatorname{LiF}(200)$, but do not include the values for Quartz(203). For this crystal we have performed calculations using the computer code DIXI developed at the Max Planck Research Unit "X-Ray Optics.' The code is based on the theoretical approach used by Taupin [24] for bent perfect crystals. Results of this calculation are shown in Fig. 2. For comparison, the values calculated by Henke, Gullikson, and Davis [23] for perfect $\mathrm{LiF}(200)$ are shown in Fig. 2 as solid circles. Calculations performed at the Max Planck Research Unit for perfect $\mathrm{LiF}(200)$ crystals agree with their numbers within $2 \%$. Figure 2 shows that despite the differences in the intrinsic structure the values of $R$ for $\operatorname{LiF}(200)$ and Quartz(203) closely track each other when displayed as a function of Bragg angle. For both crystals the values of $R$ are close to the limits set by the $|\cos (2 \theta)|$ dependence for perfect crystals. Detailed $\mathrm{X}$-ray-optical investigations have shown that quartz forms nearly perfect crystals [25] so that the use of the dynamic theory for perfect crystals is justified. Similarly, $\operatorname{LiF}(200)$ may also form nearly perfect crystals [26], although this may not always be the case. Several of the $\mathrm{LiF}(200)$ crystals used on EBIT were tested by x-ray topography at the Max Planck Research Unit. The measurements showed a network of small angle grain boundaries (average grain size is about 1 $\mathrm{cm}^{2}$, average mosaic spread is 20-60 arc sec), which is characteristic for high-quality LiF crystals. The mosaic spread increases considerably due to bending. The values of $R$ for the $\operatorname{LiF}(200)$ crystal employed in the present measurements thus may differ somewhat from the values calculated for a perfect crystal. In the following we nevertheless use the value calculated for a perfect crystal. As the analysis in the next section shows, a $10 \%$ uncertainty in the calculated values of $R$ has a negligible contribution to the overall uncertainty of our measurements. In fact, approximating $R$ by the average value for perfect and mosaic crystals changes the values inferred for the polarization by less than the uncertainty limits set by the counting statistics of the measurement. The uncertainty due to real structure defects can be avoided by using only nearly perfect crystals. For example, $\mathrm{LiF}(200)$ can be replaced by $\mathrm{Si}(220)$ or Quartz(112), which are nearly perfect crystals and have almost the same $2 d$ spacing as $\operatorname{LiF}(200)$. However, they also have a lower integrated reflectivity that results in a larger statistical uncertainty unless the measuring time is lengthened.

As seen from Fig. 2, the relative reflectivity $R$ for the Quartz(203) crystal at $\theta=42.5^{\circ}$ is $R_{\text {Qrtz }}=0.04$. In the following, we approximate this value by zero without loss of precision in the inferred polarization from our measurements. The intensity measured with the Quartz(203) crystal is thus

$$
I_{\mathrm{Qrtz}}^{\mathrm{obs}}=R_{\|} I_{\|} \text {. }
$$

The ratio of two lines $a$ and $b$ observed with the quartz crystal is then given by

$$
\left.\frac{I^{a}}{I^{b}}\right|_{\mathrm{Qrtz}}=\frac{I_{\|}^{a}}{I_{\|}^{b}} .
$$

Here we assume that $R_{\|}$and $R_{\perp}$ stay constant in the range of Bragg angles spanned by the two lines. For the $\operatorname{LiF}(200)$ crystal, $R_{\mathrm{LiF}}=0.56$ at $\theta=27.5^{\circ}$. The ratio of two lines observed with the LiF crystal is therefore

$$
\left.\frac{I^{a}}{I^{b}}\right|_{\mathrm{LiF}}=\frac{I_{\|}^{a}+R_{\mathrm{LiF}} I_{\perp}^{a}}{I_{\|}^{b}+R_{\mathrm{LiF}} I_{\perp}^{b}} .
$$

Making use of Eq. (1), which we can express as

$$
\frac{I_{\|}}{I_{\perp}}=\frac{1+P}{1-P},
$$

and combing Eqs. (22) and (23), we can eliminate $I_{\|}$and $I_{\perp}$ from the equation and solve for the polarization $P_{a}$ of line $a$ in terms of polarization $P_{b}$ of the line $b$ :

$$
P_{a}=\frac{\left.\left(R_{\mathrm{LiF}}+1\right)\left(I^{a} / I^{b}\right)\right|_{\mathrm{Qrtz}}-\left.\left(I^{a} / I^{b}\right)\right|_{\mathrm{LiF}}\left\{1+R_{\mathrm{LiF}}\left[\left(1-P_{b}\right) /\left(1+P_{b}\right)\right]\right\}}{\left.\left(R_{\mathrm{LiF}}-1\right)\left(I^{a} / I^{b}\right)\right|_{\mathrm{Qrtz}}+\left.\left(I^{a} / I^{b}\right)\right|_{\mathrm{LiF}}\left\{1+R_{\mathrm{LiF}}\left[\left(1-P_{b}\right) /\left(1+P_{b}\right)\right]\right\}} .
$$


If line $b$ is unpolarized, the dependence on $P_{b}$ drops out, and $P_{a}$ can be directly determined from the measured line ratios. The procedure can thus be applied to the measurement of a wide variety of cases where unpolarized lines are present in the spectrum. Notable examples are the $K$-shell spectra of hydrogenlike and lithiumlike ions, where transitions from upper levels with total angular momentum $J=\frac{1}{2}$, such as the Lyman- $\alpha_{2}$ line, provide excellent unpolarized reference lines. The fact that $P$ strictly vanishes for any line that proceeds from a level with total angular momentum $J=\frac{1}{2}$ follows from the properties of the Wigner 3-j symbols, where $B_{\lambda \geqslant 2}=0$ for lines with $J_{i}=\frac{1}{2}$ [cf. Eq. (4)], from the properties of the associated Legendre function, where $P_{0}^{2}=0$ for any argument, and from the properties of the 6- $j$ symbols, where $U_{\lambda \geqslant 2}=0$ for $J_{f}=\frac{1}{2}$ [cf. Eq. (11)]. No unpolarized line exists among the heliumlike Fe XXV transitions, and the value of $P_{a}$ can, in principle, only be determined from the measured line intensities, if $P_{b}$ is known. Although the polarization of none of the lines in heliumlike Fe XXV is known a priori, we can make use of the fact that the polarization of line $z$ is completely determined by that of line $x$ [cf. Eq. (19)]. Thus we use an iterative procedure to solve Eq. (25) for $P_{x}$ and $P_{z}$ by first calculating $P_{x}$ from the measured intensities assuming $P_{z}=0$. We then use the value calculated for $P_{x}$ to calculate a new estimate for $P_{z}$, which in turn is used to calculate a new value for $P_{x}$, and so on. This procedure converges within a few iterations. Once the polarizations $P_{x}$ and $P_{z}$ are known, the values for all other lines can be computed directly from Eq. (25).

\section{MEASUREMENT}

The measurements were carried out at the Livermore EBIT facility $[9,10]$, which has been used for a wide variety of measurements of the x-ray line emission from highly charged ions $[27,28]$. For the present measurement we employed the EBIT Bragg crystal spectrometer described in Ref. [29]. The instrument utilizes the focusing geometry developed by von Hámos [30]. It monitors and analyzes radiation emitted in the plane perpendicular to the electron beam, as illustrated in Fig. 3. The iron $K \alpha$ spectrum obtained with the Quartz(203) crystal is shown in Fig. 4(a). The crystal was bent to a radius of curvature $R_{c}=120 \mathrm{~cm}$, affording a nominal instrumental resolving power of $\lambda / \Delta \lambda=13000$. The observed emission lines were broadened by thermal Doppler broadening [31] and were wider than the instrumental width. The data were accumulated in a set of spectra that comprised a total of $14 \mathrm{~h}$ of observation time at a beam current $I=168$ $\mathrm{mA}$ and a beam energy $E=6.8 \mathrm{keV}$. This energy is about 100 $\mathrm{eV}$ above the threshold for excitation of the $K \alpha$ transitions. The four transitions $w, x, y$, and $z$ in Fe XXV are clearly seen. A few weaker, unlabeled lines populated by inner-shell excitation of the $1 s^{2} 2 s$ ground state of Fe XXIV and of the $1 s^{2} 2 s^{2}$ ground state of Fe XXIII are also seen. These include the transitions $1 s 2 s 2 p^{2} P_{3 / 2} \rightarrow 1 s^{2} 2 s^{2} S_{1 / 2}$ near $1.861 \AA$ and $1 s 2 s^{2} 2 p^{1} P_{1} \rightarrow 1 s^{2} s^{2}{ }^{1} S_{0}$ near $1.872 \AA$.

The spectrum of the iron $K \alpha$ transitions recorded with a $\mathrm{LiF}(200)$ crystal is shown in Fig. 4(b). The crystal was bent to a radius of curvature $R_{c}=75 \mathrm{~cm}$, affording an instrumental resolving power of $\lambda / \Delta \lambda=3400$. The LiF spectrum was obtained at a beam current $I=148 \mathrm{~mA}$ and a beam energy

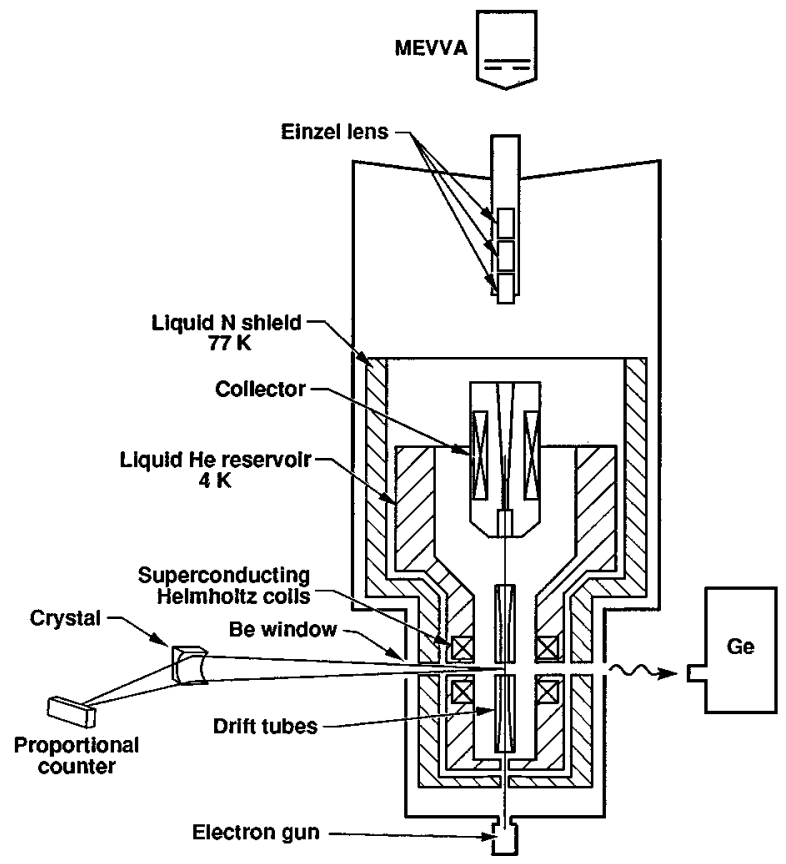

FIG. 3. Schematic layout of the von Hámos-type crystal spectrometer on EBIT. Ions are produced and trapped in a 2-cm-long region between the superconducting Helmholtz coils. The ions are excited by an electron beam propagating between the electron gun and the collector. $\mathrm{X}$ rays are monitored via the direct line-of-sight access provided by radial ports in the vacuum vessel and are analyzed and dispersed with a crystal spectrometer in a plane perpendicular to the direction of the electron beam.

$E=6.8 \mathrm{keV}$. The spectrum was accumulated over a $5 \frac{1}{2}-\mathrm{h}$ interval.

The relative intensities of the four heliumlike lines are clearly different in the two spectra. A listing of the measured intensities of the heliumlike transitions is given in Table III. Using the iterative procedure described earlier we determine $P_{w}=+0.56, P_{x}=-0.53, P_{y}=-0.22$, and $P_{z}=-0.076$, as summarized in Table II. The table also lists the $68 \%$ confidence limits of each value that have been determined based on the number of counts recorded in each line. The uncertainty resulting from the uncertainty in the respective crystal reflectivities was estimated by repeating the data reduction with values of $R$ that were $10 \%$ higher or lower than the value of 0.56 used for the LiF crystal. The resultant variations in the inferred polarizations are significantly less than the spread of values given by the statistical uncertainties and, when added in quadrature, do not change the stated uncertainties. In fact, using $R_{\mathrm{LiF}}=0.45$, which is the average value of the extremes given for a perfect and mosaic crystal, we obtain $\quad P_{w}=+0.72, \quad P_{x}=-0.56, \quad P_{y}=-0.24, \quad$ and $P_{z}=-0.080$. These values lie within the $68 \%$ confidence limits listed in Table II.

\section{DEPOLARIZATION EFFECTS}

The properties of the electron beam in EBIT can be described using the optical model developed by Herrmann $[9,32]$. According to this model, the motion of a given electron has a finite velocity component perpendicular to the $z$ axis related to the electron's radial position and velocity at 

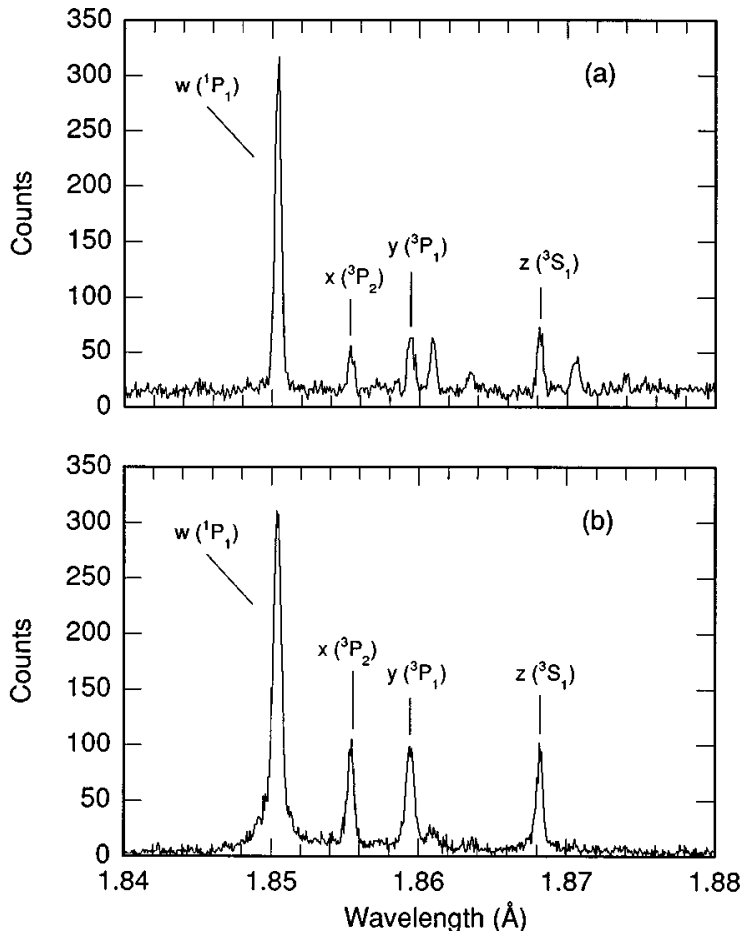

FIG. 4. Crystal-spectrometer spectra of lines $w, x, y$, and $z$ in Fe XXV excited by a $6800-\mathrm{eV}$ electron beam. (a) Spectrum obtained with a Quartz(203) crystal at a Bragg angle of $42.5^{\circ}$; (b) spectrum obtained with a $\operatorname{LiF}(200)$ crystal at a Bragg angle of $27.5^{\circ}$. Unlabeled features are from transitions in Fe XXIV and Fe XXIII formed by inner-shell excitation.

birth on the cathode of the electron gun. Herrmann showed that the magnitude of the transverse velocity is inversely proportional to the radii of the cathode images formed at various locations along the $z$ axis by the beam [32]. In other words, the product of beam area and transverse electron energy $E_{\perp}$ are constant at these locations. We can thus estimate $E_{\perp}$ from the temperature of the cathode of the electron gun $(\approx 0.1 \mathrm{eV})$ and the areal compression ratio of the beam (from a radius of about $1 \mathrm{~mm}$ at the cathode to about $0.03 \mathrm{~mm}$ in the trap) to be $E_{\perp}=110 \mathrm{eV}$. Because of the transverse component of the electron velocity, the velocity vector of a given electron and thus that of the relative electron-ion motion is no longer aligned with the $z$ axis, as assumed above. Instead, it deviates from the $z$ axis by an angle $\gamma$ given by

$$
\tan \gamma=\frac{\sqrt{E_{\perp}}}{\sqrt{E_{\text {beam }}-E_{\perp}}} .
$$

TABLE III. Intensities of the heliumlike lines $w, x, y$, and $z$ measured with a Quartz(203) and a $\operatorname{LiF}(200)$ crystal.

\begin{tabular}{lcc}
\hline \hline Line & $\begin{array}{c}\text { Quartz(203) } \\
\text { (counts) }\end{array}$ & $\begin{array}{c}\mathrm{LiF}(200) \\
\text { (counts) }\end{array}$ \\
\hline$w$ & 1740 & 4570 \\
$x$ & 191 & 1210 \\
$y$ & 292 & 1240 \\
$z$ & 284 & 1062 \\
\hline \hline
\end{tabular}

or $\gamma=7.3^{\circ}$ in the case of our measurement with $E_{\text {beam }}=6800$ $\mathrm{eV}$. The angle $\vartheta$ between the velocity vector of a given electron and the line of observation thus deviates from the value of $\vartheta=90^{\circ}$ assumed in the expressions for $I_{\perp}$ and $I_{\|}$in Eqs. (2) and (3). Instead, its value ranges between $90^{\circ}-\gamma$ and $90^{\circ}+\gamma$.

To account for arbitrary values of $\vartheta$, the arguments of the Legendre and associated Legendre polynomials need to be evaluated at $\cos \vartheta$ instead of at the origin [20]. Only even orders of the Legendre and associated Legendre polynomials occur so that the dependence on $\vartheta$ enters the modified expressions via a $\cos ^{2} \vartheta$ term. In the present case, we estimate $\cos ^{2} \vartheta \approx 0.016$ by approximating $\vartheta=90^{\circ} \pm \gamma$. The approximation used above of evaluating the Legendre and associated Legendre polynomials at the origin, thus, is justified. In fact, a calculation of the polarization of line $w$, for example, at an observation angle $\vartheta=82.7^{\circ}$ yields a value that differs from the value calculated at $\vartheta=90^{\circ}$ by merely $0.7 \%$. Depolarization effects introduced by the thermal velocity component of the electron beam are, thus, negligibly small compared to the uncertainty limits of our measurement and no corrections are made.

\section{DISCUSSION AND CONCLUSION}

A comparison between the measured polarization and theoretical values is given in Table II. The measured values agree very well within statistical confidence limits with the values predicted by the distorted-wave calculations, as illustrated in Fig. 1. By contrast, the measured values differ significantly from the values computed from Coulomb-Born cross sections $[18,19]$. Our measurements, thus, can distinguish between the two theoretical approaches and show that Coulomb-Born calculations, with the possible exception of the ${ }^{3} P_{1}$ line, do not have sufficient accuracy to predict the observed polarizations.

Our measurement also shows unequivocally that line $z$ is polarized. This clearly demonstrates that the line is excited in part by radiative cascades from the ${ }^{3} P_{2}$ level and that such cascades must be included in any analysis. In fact, the measurement of the polarization of line $z$ represents a measurement of the cascade contribution from the ${ }^{3} P_{2}$ level. Moreover, knowing this contribution and the observed intensity of line $x$, it is possible to infer the radiative branching ratio for the decay of the ${ }^{3} P_{2}$ level. Measurements along the isoelectronic sequence could thus provide a test on this branching ratio.

Measurements along the isoelectronic sequence are also needed to test the polarization dependence of line $y$. Because its polarization varies strongly with $Z$, as illustrated in Fig. 1, a single measurement does not validate predictions away from the present datum. This is in contrast to lines $x$ and $w$. These do not vary significantly as a function of $Z$ (cf. Fig. 1), and a single measurement establishes the value of their polarization over a wide range of elements.

We can compare our measured values to those measured for heliumlike Sc XX [8]. For the singlet line $w$, which is unaffected by the hyperfine mixing, the measured polarization of $0.70 \pm 0.06$ in Sc XX [8] is to within the measurement uncertainties identical to the value found in our measurement of the line $w$ in $\mathrm{Fe} \mathrm{XXV}$, as illustrated in Fig. 1. Thus our 
measurement tests and verifies across five atomic numbers the prediction that the polarization of the singlet line is virtually the same for different elements. Because of the strong hyperfine mixing among the magnetic sublevel populations of the triplet levels in Sc XX, the polarization of all triplet lines vanishes. Indeed, the values of $-0.05 \pm 0.09,0.00$ \pm 0.09 , and $-0.02 \pm 0.10$ measured for the lines $x, y$, and $z$, respectively, differ markedly from the values of $+0.56_{-0.08}^{+0.17}$, $-0.22_{-0.02}^{+0.05}$, and $-0.076_{-0.007}^{+0.007}$ observed for these lines in Fe XXV. In the case of the triplet lines, our measurement is complementary to the measurement of the polarization of the $K \alpha$ emission lines of heliumlike Sc XX and represents a test of theory in a situation where the polarization of the triplet lines of a highly charged heliumlike ion is nonvanishing.

\section{ACKNOWLEDGMENTS}

This work was supported by the National Aeronautics and Space Administration X-Ray Astronomy Research and Analysis Program under Grant No. NAGW-4185 and performed under the auspices of the Department of Energy by Lawrence Livermore National Laboratory under Contract No. W-7405-ENG-48.
[1] J. R. Oppenheimer, Z. Phys. 43, 27 (1927).

[2] I. C. Percival and M. J. Seaton, Philos. Trans. R. Soc. London, Ser. A 251, 113 (1958).

[3] R. Beier, C. Bachmann, and R. Burhenn, J. Phys. D 14, 643 (1981).

[4] J. C. Kieffer, J. P. Matte, H. Pépin, M. Chaker, Y. Beaudoin, T. W. Johnston, C. Y. Chien, S. Coe, G. Mourou, and J. Dubau, Phys. Rev. Lett. 68, 480 (1992).

[5] J. C. Kieffer, J. P. Matte, M. Chaker, Y. Beaudoin, C. Y. Chien, S. Coe, G. Mourou, J. Dubau, and M. K. Inal, Phys. Rev. E 48, 4648 (1993).

[6] E. Haug, Sol. Phys. 71, 77 (1981).

[7] J. M. Laming, Astrophys. J. 357, 275 (1990).

[8] J. R. Henderson, P. Beiersdorfer, C. L. Bennett, S. Chantrenne, D. A. Knapp, R. E. Marrs, M. B. Schneider, K. L. Wong, G. A. Doschek, J. F. Seely, C. M. Brown, R. E. La Villa, J. Dubau, and M. A. Levine, Phys. Rev. Lett. 65, 705 (1990).

[9] M. A. Levine, R. E. Marrs, J. R. Henderson, D. A. Knapp, and M. B. Schneider, Phys. Scr. T22, 157 (1988).

[10] M. A. Levine, R. E. Marrs, J. N. Bardsley, P. Beiersdorfer, C. L. Bennett, M. H. Chen, T. Cowan, D. Dietrich, J. R. Henderson, D. A. Knapp, A. Osterheld, B. M. Penetrante, M. B. Schneider, and J. H. Scofield, Nucl. Instrum. Methods Phys. Res. Sect. B 43, 431 (1989).

[11] M. K. Inal and J. Dubau, J. Phys. B 20, 4221 (1987).

[12] Y. Itikawa, R. Srivastava, and K. Sakimoto, Phys. Rev. A 44, 7195 (1993).

[13] M. K. Inal and J. Dubau, Phys. Rev. A 47, 4794 (1993).

[14] K. J. Reed and M. H. Chen, Phys. Rev. A 48, 3644 (1993).

[15] I. A. Zhitnik, V. V. Korneev, V. V. Krutov, S. N. Oparin, and A. M. Ubnov, in X-Ray Spectroscopy and Properties of Multiply Charged Ions, Proceedings of the Lebedev Physics Institute Vol. 179, edited by I. E. Sobelman (Nova Science, Commack, NY, 1988), p. 51.

[16] A. H. Gabriel, Mon. Not. R. Astron. Soc. 160, 99 (1972).

[17] C. D. Lin, W. R. Johnson, and A. Dalgarno, Phys. Rev. A 15, 154 (1977).

[18] A. S. Shlyaptseva, A. M. Urnov, and A. V. Vinogradov, P. N. Lebedev Physical Institute of the USSR Academy of Sciences
Report No. 194, 1981 (unpublished).

[19] A. V. Vinogradov, A. M. Urnov, and A. S. Shlyaptseva, in Atomic and Ionic Spectra and Elementary Processes in Plasma, Proceedings of the Lebedev Physics Institute Academy of Sciences of Russia Vol. 192, edited by I. I. Sobelman (Nova Science, Commack, NY, 1992), p. 93.

[20] R. M. Steffen and K. Alder, in The Electromagnetic Interaction in Nuclear Spectroscopy, edited by W. D. Hamilton (North-Holland, New York, 1975), p. 505.

[21] H. L. Zhang, D. H. Sampson, and R. E. H. Clark, Phys. Rev. A 41, 198 (1990).

[22] A. Burek, Space Sci. Instrum. 2, 53 (1976).

[23] B. L. Henke, E. M. Gullikson, and J. C. Davis, At. Data Nucl. Data Tables 54, 181 (1993).

[24] D. Taupin, Bull. Soc. Fr. Mineral. Cristallogr. 87, 469 (1964).

[25] E. Förster, J. Heinisch, P. Heist, G. Hölzer, I. Uschmann, F. Scholze, and F. Schäfers, in BESSY Jahrbuch (annual report), 1992, p. 481 (unpublished).

[26] N. G. Alexandropoulos and G. G. Cohen, Appl. Spectrosc. 28, 155 (1974).

[27] P. Beiersdorfer, R. Cauble, S. Chantrenne, M. H. Chen, N. DelGrande, D. Knapp, R. E. Marrs, A. L. Osterheld, K. Reed, M. Schneider, J. Scofield, B. Wargelin, D. A. Vogel, K. Wong, and R. Zasadzinski, in $U V$ and X-Ray Spectroscopy of Astrophysical and Laboratory Plasmas, edited by E. Silver and S. Kahn (Cambridge University Press, Cambridge, England, 1993), p. 59.

[28] P. Beiersdorfer, M. Bitter, M. Chen, V. Decaux, S. Elliott, S. Kahn, D. Knapp, R. Marrs, A. Osterheld, D. Vogel, and K. Widmann, in Atomic Processes in Plasmas, edited by W. L. Rowan, AIP Conf. Proc. No. 322 (AIP, New York, 1995), p. 129.

[29] P. Beiersdorfer, R. E. Marrs, J. R. Henderson, D. A. Knapp, M. A. Levine, D. B. Platt, M. B. Schneider, D. A. Vogel, and K. L. Wong, Rev. Sci. Instrum. 61, 2338 (1990).

[30] L. v. Hámos, Ann. Phys. (Leipzig) 17, 716 (1933).

[31] P. Beiersdorfer, V. Decaux, S. Elliott, K. Widmann, and K. Wong, Rev. Sci. Instrum. 66, 303 (1995).

[32] G. Herrmann, J. Appl. Phys. 29, 127 (1958). 